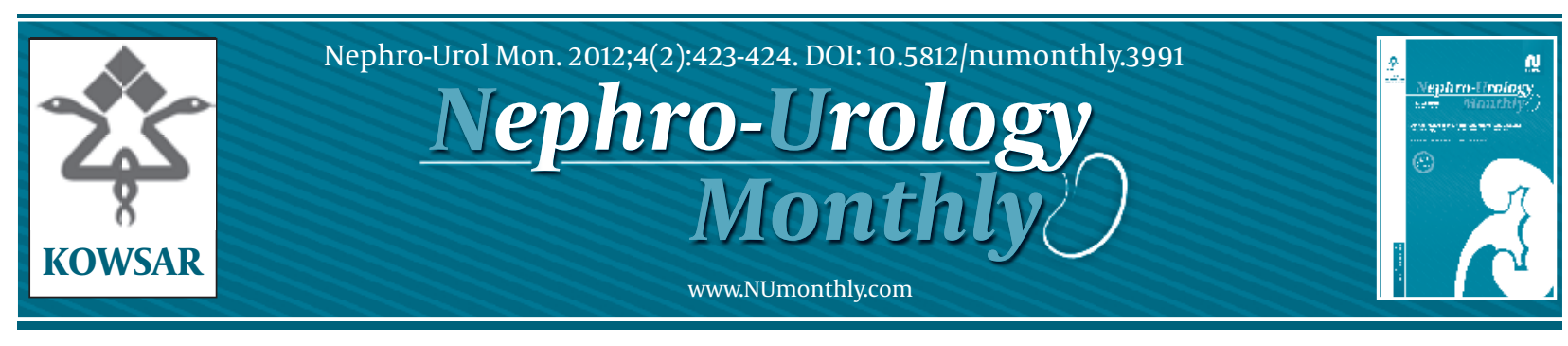

\title{
To Biopsy a Small Renal Mass, or Not?
}

\author{
Elefterios Chatzidarellis ${ }^{1}$, Andreas Skolarikos ${ }^{1}$, Athanasios G Papatsoris ${ }^{1^{*}}$ \\ ${ }^{1}$ Department of Urology, School of Medicine, University of Athens, Sismanoglio Hospital, Athens, Greece
}

A R T I C L E I N F O

Article type:

Editorial

Article history:

Received: 26 Dec 2011

Revised: 01 Jan 2012

Accepted: 07 Jan 2012

Keywords:

Biopsy

Kidney

The abundant use of cross-sectional imaging has markedly increased the diagnosis of small renal masses (SRMs) (1). A strict definition of small renal masses is lacking and most authors consider lesions $<4 \mathrm{~cm}$ as small (2). According to the literature, $20 \%$ of SRMs are benign in formal histological results and SRMs represent $48-66 \%$ of diagnosed renal cell cancers (RCC) $(1,2)$. In a meta-analysis by Chawla et al. (3) the incidence of metastasis of SRMs is $1 \%$ after a 3-year follow up. Nevertheless, the radiological features of SRMs are insufficient to describe the biological potential and predict the natural history of SRMs (4). Therefore, studies have demonstrated overtreatment of patients with benign SRMs which were misdiagnosed as malignant on the preoperative imaging $(2,4)$. The inability of modern imaging techniques (i.e. computerized tomography (CT) to differentiate benign from malignant SRMs has renewed interest in renal mass biopsy (RMB). Furthermore, the renewed interest in RMB is closely associated with novel minimal invasive treatments for SRMs (i.e. cryotherapy and radiofrequency ablation (RFA), improved biopsy techniques and pathological evaluation (5).

RMB aims to determine eventual malignancy, type and grade of the evaluated SRM with high specificity and sensitivity for the presence of malignancy. The diagnostic

* Corresponding author: Athanasios G Papatsoris, Department of Urology, School of Medicine, University of Athens, Sismanoglio Hospital, Athens, Greece. Tel: +30-2108039253, Fax: +30-2108044703, E-mail: agpapatsoris@ yahoo.gr

DOI:10.5812/numonthly.3991

Copyright $\odot 2012$ Kowsar M. P. Co. All rights reserved.
Implication for health policy/practice/research/medical education:

As abdominal imaging has increased the diagnosis of small renal masses (SRMs) the role of renal mass biopsy (RMB) is debatable. Current literature demonstrates that RMB is recommended for the diagnosis, follow-up surveillance and ablative therapies of SRMs.

\section{- Please cite this paper as:}

Chatzidarellis E, Skolarikos A, Papatsoris AG. To Biopsy a Small Renal Mass or Not? Nephro-Urol Mon. 2012;4(2): 423-4. DOI: 10.5812/numonthly.3991

Copyright $\odot 2012$ Kowsar M. P. Co. All rights reserved.

accuracy of RMB has improved through the last years, as has been demonstrated in a recent meta-analysis by Lane et al. (5) The authors compared the diagnostic accuracy of RMB with reports stratified pre- and post-2001, showing an improvement from $88 \%$ to $94 \%$. The diagnostic value of RMB refers not only to the differential diagnosis of benign versus malignant tumors, but also to the definition of the histological subtype of the tumor (i.e. renal cell carcinoma (RCC). Neuzillet et al. (6) showed high concordance (more than 91\%), between the histological subtype findings of the RMB and the final nephrectomy specimen for SRMs (8).

The recent evolution of minimally invasive treatment options for SRMs highlights even more the important role of the RMB, before cryotherapy or RFA where no biopsy is possible (5). CT guided RMB has been demonstrated to improve the differential diagnosis of SRMs and prevent overtreatment (7). Furthermore, RMB can nowadays change the management of SRM which was doubtful a few years ago (8). A benign or SRM of low malignant potential can be managed with active surveillance protocols or ablative techniques especially in elderly and unfit patients. Moreover, the positive predictive value of imaging findings is so high that a negative RMB does not alter management (5). Furthermore, RMB is indicated in metastatic patients, before starting systemic therapy (7). In recent studies, RMB-related complications such as gross hematuria, renal hematoma requiring intervention (i.e. admission, transfusion or nephrectomy), arterio-venous fistula or pneumothorax are extremely rare $(<1 \%)(9)$. 
The traditional concern of needle tract tumor seeding is based on only six reports published before 1993 (5). The majority of these cases were transitional cell carcinoma of the upper urinary tract, which is a contraindication for RMB. Interestingly, since 1993 no case of tumor seeding has been reported, most probably because of the innovative needle introducers which isolate the sample from the surrounding tissues.

Repeat RMB is suggested in cases of a non-diagnostic result due to an inadequate or insufficient specimen (9). In order to reduce the incidence of such non-diagnostic RMB there are several recommendations. RMB guided by CT and/or real time ultrasound increases diagnostic accuracy (10). Two cores of $15-22 \mathrm{~mm}$ in length each taken by an 18-gauge biopsy gun provide reliable specimens (11). Furthermore, by targeting the peripheral area of the SRM, potential central necrosis is avoided. Moreover, by placing the tip of the needle a few millimeters outside the SRM, the specimen includes the capsule of the tumor. New molecular techniques such as, polymerase chain reaction and fluorescence in situ hybridization, improve the accuracy of tumor sub-typing (12). Moreover, modern immunocytochemistry can contribute to the differential diagnosis between RCC and several benign tumors, such as oncocytomas (13).

Nowadays, RBM has become an established tool for the management of SRMs as indicated in the recent European Association of Urology (EAU) guidelines (14). It is recommended for the diagnosis, follow-up surveillance and ablative therapies of SRMs. The future looks promising for RMB in the evaluation of SRMs, especially with the implications of molecular and cytogenetic profiling of the specimen. Eventually, RMB might be able to predict the prognosis of the SRM and guide its treatment. There is no easy answer to the difficult question, to biopsy a SRM or not, however the trend will be to continue to perform the biopsy.

\section{Financial Disclosure}

There is no finacial disclosure.

\section{References}

1. Cooperberg MR, Mallin K, Ritchey J, Villalta JD, Carroll PR, Kane CJ. Decreasing size at diagnosis of stage 1 renal cell carcinoma: analysis from the National Cancer Data Base, 1993 to 2004.J Urol. 2008;179(6):2131-5.

2. Volpe A, Panzarella T, Rendon RA, Haider MA, Kondylis FI, Jewett MA. The natural history of incidentally detected small renal masses. Cancer. 2004;100(4):738-45.

3. Chawla SN, Crispen PL, Hanlon AL, Greenberg RE, Chen DY, Uzzo RG. The natural history of observed enhancing renal masses: meta-analysis and review of the world literature. J Urol. 2006;175(2):425-31.

4. Remzi M, Katzenbeisser D, Waldert M, Klingler HC, Susani M, Memarsadeghi M, et al. Renal tumour size measured radiologically before surgery is an unreliable variable for predicting histopathological features: benign tumours are not necessarily small. BJU Int. 2007;99(5):1002-6.

5. Lane BR, Samplaski MK, Herts BR, Zhou M, Novick AC, Campbell SC. Renal mass biopsy--a renaissance? J Urol. 2008;179(1):20-7.

6. Neuzillet Y, Lechevallier E, Andre M, Daniel L, Coulange C. Accuracy and clinical role of fine needle percutaneous biopsy with computerized tomography guidance of small (less than $4.0 \mathrm{~cm}$ ) renal masses. JUrol. 2004;171(5):1802-5.

7. Remzi M, Marberger M. Renal tumor biopsies for evaluation of small renal tumors: why, in whom, and how? Eur Urol. 2009;55(2):359-67.

8. Maturen KE, Nghiem HV, Caoili EM, Higgins EG, Wolf JS, Jr., Wood DP, Jr. Renal mass core biopsy: accuracy and impact on clinical management. AJR Am J Roentgenol. 2007;188(2):563-70.

9. Leveridge MJ, Finelli A, Kachura JR, Evans A, Chung H, Shiff DA, et al. Outcomes of small renal mass needle core biopsy, nondiagnostic percutaneous biopsy, and the role of repeat biopsy. Eur Urol. 2011;60(3):578-84.

10. Schmidbauer J, Remzi M, Memarsadeghi M, Haitel A, Klingler HC, Katzenbeisser D, et al. Diagnostic accuracy of computed tomography-guided percutaneous biopsy of renal masses. Eur Urol.2008;53(5):1003-11.

11. Breda A, Treat EG, Haft-Candell L, Leppert JT, Harper JD, Said J, et al. Comparison of accuracy of 14-, 18- and 20-G needles in exvivo renal mass biopsy: a prospective, blinded study. BJU Int. 2010;105(7):940-5.

12. Barocas DA, Mathew S, DelPizzo JJ, Vaughan ED, Jr., Sosa RE, Fine $\mathrm{RG}$, et al. Renal cell carcinoma sub-typing by histopathology and fluorescence in situ hybridization on a needle-biopsy specimen. BJU Int. 2007;99(2):290-5.

13. Barocas DA, Rohan SM, Kao J, Gurevich RD, Del Pizzo JJ, Vaughan ED, Jr., et al. Diagnosis of renal tumors on needle biopsy specimens by histological and molecular analysis. J Urol. 2006;176(5):1957-62.

14. Ljungberg B, Cowan NC, Hanbury DC, Hora M, Kuczyk MA, Merseburger AS, et al. EAU guidelines on renal cell carcinoma: the 2010 update. Eur Urol. 2010;58(3):398-406. 